\title{
Adaptive Synchronization in Complex Network with Different Order Node Dynamics
}

\author{
Fan-di Zhang, Jin-ping Jia, and Li-xin Yang \\ School of Mathematic and Statistics, Tianshui Normal University, Tianshui 741001, China \\ Correspondence should be addressed to Li-xin Yang; y0931l@163.com
}

Received 21 November 2014; Accepted 17 March 2015

Academic Editor: Luca Guerrini

Copyright (C) 2015 Fan-di Zhang et al. This is an open access article distributed under the Creative Commons Attribution License, which permits unrestricted use, distribution, and reproduction in any medium, provided the original work is properly cited.

\begin{abstract}
This paper investigates the adaptive cluster synchronization in the complex networks with different orders. By means of Lyapunov stability theory and the adaptive control technique, a novel adaptive synchronization controller is developed. To demonstrate the validity of the proposed method, the examples for the synchronization of systems with the chaotic and hyperchaotic node dynamics are presented.
\end{abstract}

\section{Introduction}

In the past few years, complex networks have attracted more and more attention; examples of complex networks include the Internet, social networks, biological networks, organizational networks, neural networks, and many others [1-6].

A complex network can be seen as a large set of interconnected nodes and used to describe various systems with specific contents. Synchronization constitutes one of the most prevalent collective dynamics in complex networked systems. Until now, several types of synchronization have been investigated, such as phase synchronization and complete synchronization [7-9], projective synchronization [10-13], and function projective synchronization [14].

In general, there are two main factors devoting to network synchronization. One is the isolated node dynamics, and the other is the complicated network topology. In almost all the existing literature, a common assumption is that all nodes are identical. It should be noted that complex networks consisting of different nodes are ubiquitous in various fields. In the present paper, we focus on cluster synchronization where the local dynamics of the nodes in each group differ. Here by a group we mean a collection of systems that have the same dynamics, with any given group consisting of systems with dynamics that is different from the dynamics of systems in the other groups.
Specifically, in many technological, social, and biological networks, which can be divided naturally into several groups by certain rules, nodes in the same group often have the same type of function. The phenomenon of cluster synchronization is observed when an ensemble of oscillators splits into groups of synchronized elements. Up to now, much research effort has been devoted to investigating the cluster synchronization of complex dynamical networks $[15,16]$. In [17], researchers studied the cluster synchronization for directed community networks via pinning partial schemes. However, the cluster synchronization where the local dynamics of the nodes in each group differ is a more interesting topic. To date, very little research effort has been done about the generalization cluster synchronization.

The organization of this paper is organized as follows. Model of complex network with community structure is given in Section 2. In Section 3, based on the Lyapunov stability theory, an adaptive nonlinear controller is developed for synchronization of complex networks with different orders. Section 4 shows the validity of the proposed synchronization scheme through numerical simulations. Finally, the conclusions of this paper are drawn in Section 5.

\section{Model Description}

Consider a dynamical network with $M$ community of coupled chaotic oscillators; also the local dynamics can be 


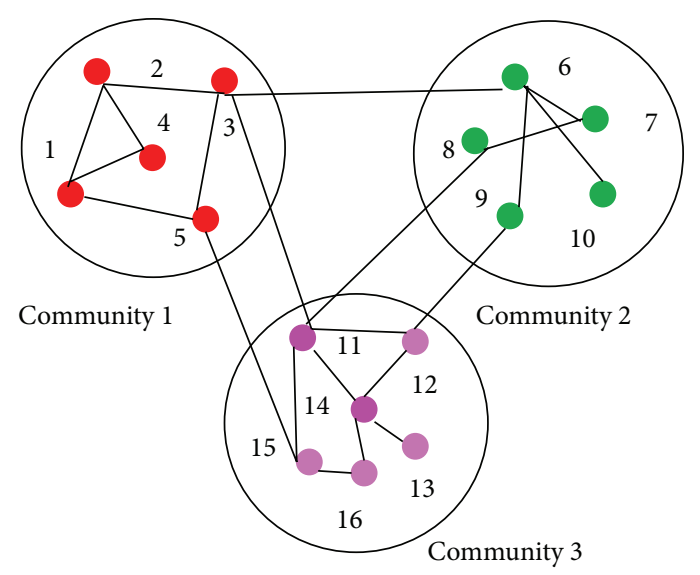

FIGURE 1: Topology structure of the complex networks with three communities.

different for each community but must be identical for all nodes in the same community. Suppose that the $k$ th community is composed of $N_{k}$ nodes. The dynamical variables of the nodes in each community are then given by $x_{i}^{k} \in R^{d_{k}}$.

For better describing this kind of community network, a community network model with different orders is introduced. Figure 1 shows topology structure of the complex networks with three communities.

A $M$-communities-network consists of $N$ nodes, which can be described by the differential equation:

$$
\begin{array}{r}
\dot{x}_{i}(t)=F^{(k)}\left(x_{i}^{k}(t)\right)+\sum_{j=1, i \neq j}^{N_{k}} a_{i j}(t) H_{k k}\left(x_{j}(t)-x_{i}(t)\right) \\
+\sum_{p=1, p \neq k}^{M} \sum_{j=1}^{N_{p}} a_{i j}(t)\left(H_{k p} x_{j}(t)-H_{k k}^{p} x_{i}(t)\right) \\
i=1,2, \ldots, N_{k}, k=1,2, \ldots, M,
\end{array}
$$

where $x_{i}^{k}=\left(x_{i 1}^{k}, x_{i 2}^{k}, \ldots, x_{i n_{k}}^{k}\right)^{T} \in R^{n_{k}}$ is the state vector of the $i$ th node that belongs to the $k$ th community. $F_{k}$ is a smooth nonlinear function vector which describes the node's dynamics of the $k$ th community. The matrix $H_{k k}=$ $\operatorname{diag}\left(h_{k k}^{1}, h_{k k}^{2}, \ldots, h_{k k}^{n_{k}}\right)$ is the inner coupling matrix which represents the internal connection in $k$ th community, $H_{k p}=$ $\left(h_{k p}^{r l}\right) \in R^{n_{k} \times n_{p}}$ and $H_{k k}^{p}=\operatorname{diag}\left(h_{k k}^{p(1)}, h_{k k}^{p(2)}, \ldots, h_{k k}^{\left(n_{k}\right)}\right)$ are the inner coupling matrix between the $k$ th and $p$ th communities, where if the $r$ th element of $x_{i}(t)$ in the $k$ th community is affected by the $l$ th element of $x_{j}(t)$ in the $p$ th, and then $h_{k k}^{p(r)} \neq 0, h_{k p}^{r l} \neq 0$; otherwise $h_{k k}^{p(r)}=0, h_{k p}^{r l}=0$. The matrix $A=\left(a_{i j}\right)_{N \times N}$ is the zero-row-sum outer coupling matrix and represents the network topology; if there is a connection between node $i$ and $j$ is connected, then $a_{i j}=a_{j i}>0(i \neq j)$; otherwise, $a_{i j}=a_{j i}=0(i \neq j)$.

Without loss of generality, the sets of subscripts of these clusters are $G_{1}=\left\{1,2, \ldots, N_{1}\right\}, G_{2}=\left\{N_{1}+1, N_{1}+2, \ldots, N_{1}+\right.$ $\left.N_{2}\right\}, \ldots$, and $G_{M}=\left\{N_{1}+\cdots+N_{M-1}+1, \ldots, N\right\}$, where
$N_{1}+N_{2}+\cdots+N_{M}=N$. The coupling matrix $A$ can be described as the following block form:

$$
A=\left[\begin{array}{cccc}
A_{11} & A_{12} & \cdots & A_{1 M} \\
A_{21} & A_{22} & \cdots & A_{2 M} \\
\vdots & & \ddots & \vdots \\
A_{M 1} & & & A_{M M}
\end{array}\right],
$$

where $A_{u u} \in R^{r_{u} \times r_{u}}(u=1,2, \ldots, M)$ represents the connections in the community and $A_{u v} \in R^{r_{u} \times r_{v}}(u=$ $1,2, \ldots, M, v=1,2, \ldots, M, u \neq v)$ denotes the connections between and communities. When the control inputs $u_{i}(t) \epsilon$ $R^{n}$ are introduced and the controlled dynamical network can be written as

$$
\begin{array}{r}
\dot{x}_{i}(t) \\
=F^{(k)}\left(x_{i}^{k}(t)\right)+\sum_{j=1, i \neq j}^{N_{k}} a_{i j}(t) H_{k k}\left(x_{j}(t)-x_{i}(t)\right) \\
+\sum_{l=1, l \neq k}^{M} \sum_{j=1}^{N_{l}} a_{i j}(t)\left(H_{k l} x_{j}(t)-H_{k k}^{l} x_{i}(t)\right)+u_{i}(t) \\
\quad i=1,2, \ldots, N_{k}, k=1,2, \ldots, M .
\end{array}
$$

Let the synchronous errors of the $k$ th community as following:

$$
e_{i}^{k}=x_{i}^{k}-s_{k} \quad\left(i=1,2, \ldots, N_{k}\right),
$$

where $s_{k}(t)$ is a solution of an isolated node in the $k$ th and satisfies $\dot{s}_{k}(t)=F_{k}\left(s_{k}(t)\right)$. The network achieves the cluster synchronization, if $\lim _{t \rightarrow \infty}\left\|e_{i}(t)\right\|=0, i=1,2, \ldots, N$.

\section{Synchronization Scheme}

First, we will present some useful assumptions for deriving the main results.

Assumption 1. Each block matrix $A_{u v}(u, v=1,2 \ldots, M)$ in (2) is zero row sum matrix. 
Assumption 2. Suppose that there exist nonnegative constants $\varepsilon_{i}(i=1,2, \ldots, N)$, such that for any time varying vectors $x(t), y(t) \in R^{n}$, and $\left\|F_{i}(y(t))-F_{i}(x(t))\right\| \leq \varepsilon_{i} \| y(t)-$ $x(t) \|$, where $\|\cdot\|$ is the norm of vector.

In the following, we introduce a scheme to achieve the cluster synchronization in colored network with community structure and adaptive coupling strengths. According to the above definition of the error variables and suppose that Assumption 2 holds, one can obtain

$$
\begin{aligned}
\sum_{j=1}^{N_{l}} a_{i j} H_{k l} s_{l}(t)= & \sum_{j=1}^{N_{l}} a_{i j} H_{k k}^{l} x_{i}(t)=0, \\
& \quad i=1, \ldots, N_{k}, k, l=1, \ldots, M, k \neq l .
\end{aligned}
$$

So the error dynamical system as follows:

$$
\begin{aligned}
e_{i}(t)= & F_{k}\left(x_{i}(t)\right)-F_{k}\left(s_{k}(t)\right)+\sum_{j=1}^{N_{k}} a_{i j} H_{k k} e_{j}(t) \\
& +\sum_{l=1, l \neq k}^{M} \sum_{j=1}^{N_{l}} a_{i j} H_{k l} e_{j}(t)+u_{i}(t) .
\end{aligned}
$$

In order to achieve cluster synchronization in the colored network (3), the controller is designed as follows via pinning control:

$$
\begin{aligned}
& u_{i}(t)=-\sum_{j=1}^{N} a_{i j} H_{k k} s_{j}(t)-q_{i} e_{i}(t) \\
& \dot{a}_{i j}(t)=\beta\left\|\left(x_{j}(t)-s_{j}(t)\right)-\left(x_{i}(t)-s_{i}(t)\right)\right\|,
\end{aligned}
$$

where $\beta>0, q_{i}>0$ are the adaptive gain, and feedback gain, respectively.

Theorem 3. Suppose that Assumption 1 holds; the cluster synchronization can be realized under controller (7).

Proof. Consider the following Lyapunov function:

$$
V(t)=\frac{1}{2} \sum_{i=1}^{N} e_{i}(t)^{T} e_{i}(t)+\frac{1}{2 \beta} \sum_{\xi}\left(c_{i j}-a_{i j}\right)^{T}\left(c_{i j}-a_{i j}\right) .
$$

The derivative of $V(t)$ along the trajectories of (7) can be calculated as follows:

$$
\begin{aligned}
\dot{V} & =\sum_{i=1}^{N} e_{i}(t)^{T} \dot{e}_{i}(t)+\frac{1}{\beta} \sum_{\xi}\left(c_{i j}-a_{i j}\right)^{T} \dot{a}_{i j}=\sum_{k=1}^{M} \sum_{i=1}^{N_{k}} e_{i}^{T}(t) \\
& \cdot\left[F_{k}\left(x_{i}(t)\right)\right. \\
& \left.-F_{k}\left(s_{i}(t)\right)+\sum_{j=1}^{N} a_{i j} H_{k k} e_{j}(t)-q_{i} H_{k k}\left(x_{i}-s_{k}(t)\right)\right]
\end{aligned}
$$

$$
\begin{aligned}
& -\sum_{\xi}\left(c_{i j}-a_{i j}\right)^{T}\left\|\left(x_{j}(t)-s_{j}(t)\right)-\left(x_{i}(t)-s_{i}(t)\right)\right\| \\
& \leq \varepsilon_{k} \sum_{k=1}^{M} \sum_{i=1}^{N_{k}} e_{i}^{T}(t) e_{i}(t)+\sum_{k=1}^{M} \sum_{i=1}^{N_{k}} e_{i}^{T}(t)\left[\sum_{j=1}^{N} a_{i j} H_{k k} e_{j}(t)\right. \\
& \left.-q_{i} H_{k k} e_{i}(t)\right] \\
& -\sum_{\xi}\left(c_{i j}-a_{i j}\right)^{T}\left\|\left(x_{j}(t)-s_{j}(t)\right)-\left(x_{i}(t)-s_{i}(t)\right)\right\| \\
& =\sum_{k=1}^{M} \sum_{i=1}^{N_{k}} w_{k} e_{i}^{T}(t) e_{i}(t)+\sum_{i=1}^{n} \gamma_{j} \widetilde{e}_{j}^{T}(t) A \widetilde{e}_{j}(t)-\sum_{i=1}^{n} \gamma_{j} \\
& \cdot \tilde{e}_{j}^{T}(t) Q \widetilde{e}_{j}(t) \\
& -\sum_{\xi}\left(c_{i j}-a_{i j}\right)^{T}\left\|\left(x_{j}(t)-s_{j}(t)\right)-\left(x_{i}(t)-s_{i}(t)\right)\right\| \\
& \leq \sum_{i=1}^{n} \widetilde{e}_{j}^{T}(t)\left(\varepsilon_{k} I_{N}+\gamma_{j}(A-Q)\right) \widetilde{e}_{j}(t) \\
& -\sum_{\xi}\left(c_{i j}-a_{i j}\right)^{T}\left\|\left(x_{j}(t)-s_{j}(t)\right)-\left(x_{i}(t)-s_{i}(t)\right)\right\| .
\end{aligned}
$$

For any edge $(i, j) \in \xi$, there exists a positive constant $c_{i j}$ larger than the corresponding coupling strength $a_{i j}$; that is,

$$
\sum_{\xi}\left(c_{i j}-a_{i j}\right)^{T}\left\|e_{j}(t)-e_{i}(t)\right\| \geq 0 .
$$

Also, one can choose sufficiently large positive constants $q_{i}$; that is, $\dot{V}<0$.

\section{Numerical Simulations}

Consider the community network shown in Figure 1 as an example to illustrate the effectiveness of the derived results. Choose the node dynamics of the first community as the following hyperchaotic Chen system:

$$
\begin{aligned}
& \dot{x}_{i 1}=35\left(x_{i 2}-x_{i 1}\right)+x_{i 4} \\
& \dot{x}_{i 2}=7 x_{i 1}+12 x_{i 2}-x_{i 1} x_{i 3} \\
& \dot{x}_{i 3}=x_{i 1} x_{i 2}-3 x_{i 3} \\
& \dot{x}_{i 4}=0.5 x_{i 4}+x_{i 2} x_{i 3},
\end{aligned}
$$

with $i=1,2, \ldots, 5$. 


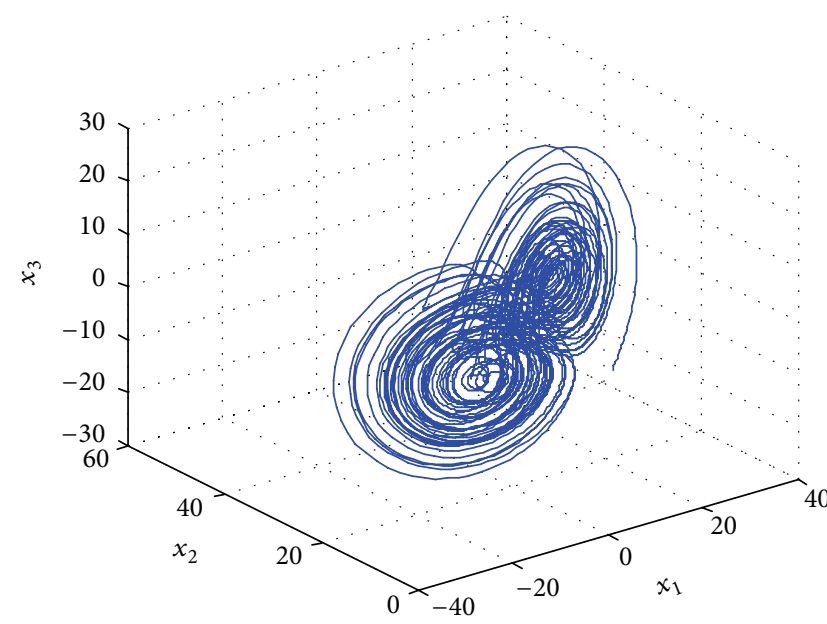

(a)

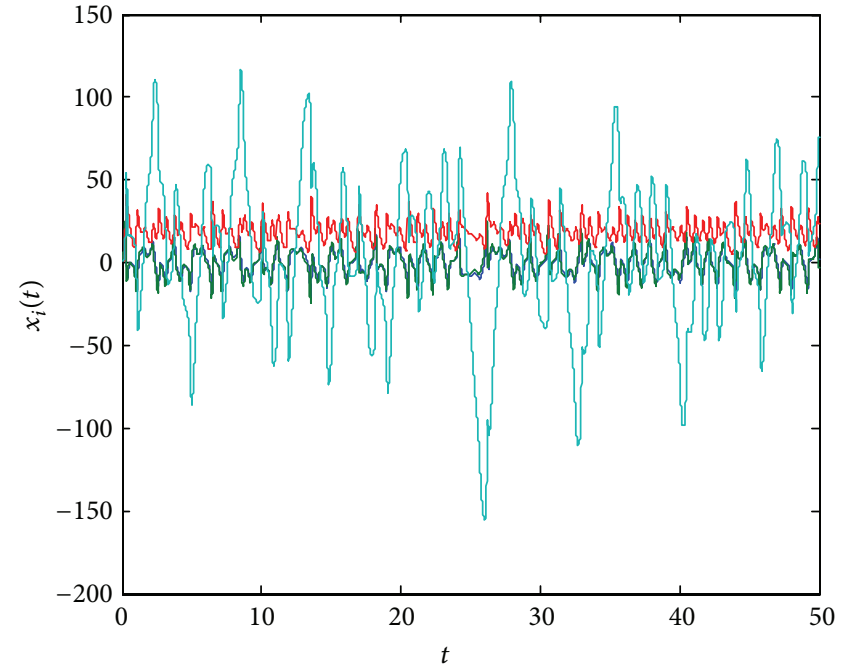

(b)

FIGURE 2: The node's dynamic in the first community.

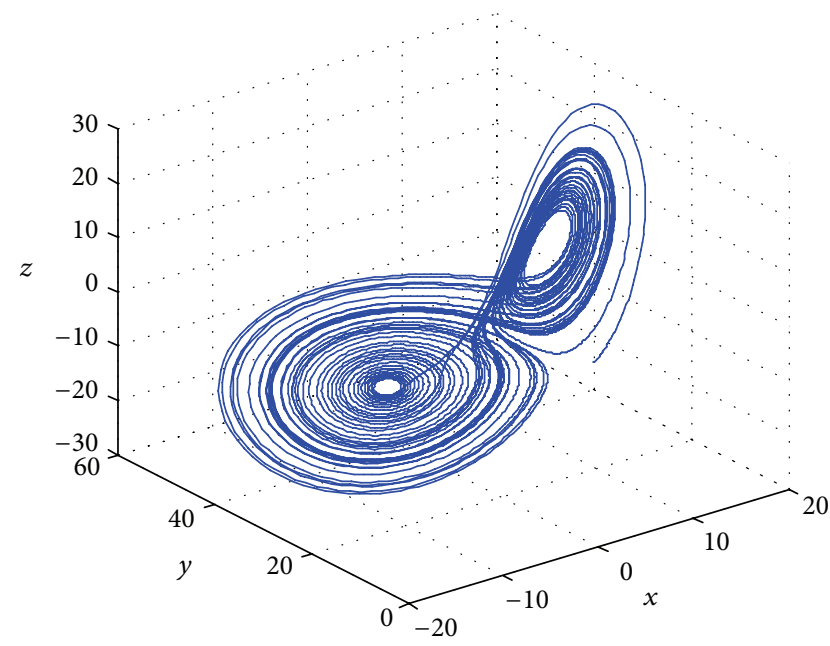

(a)

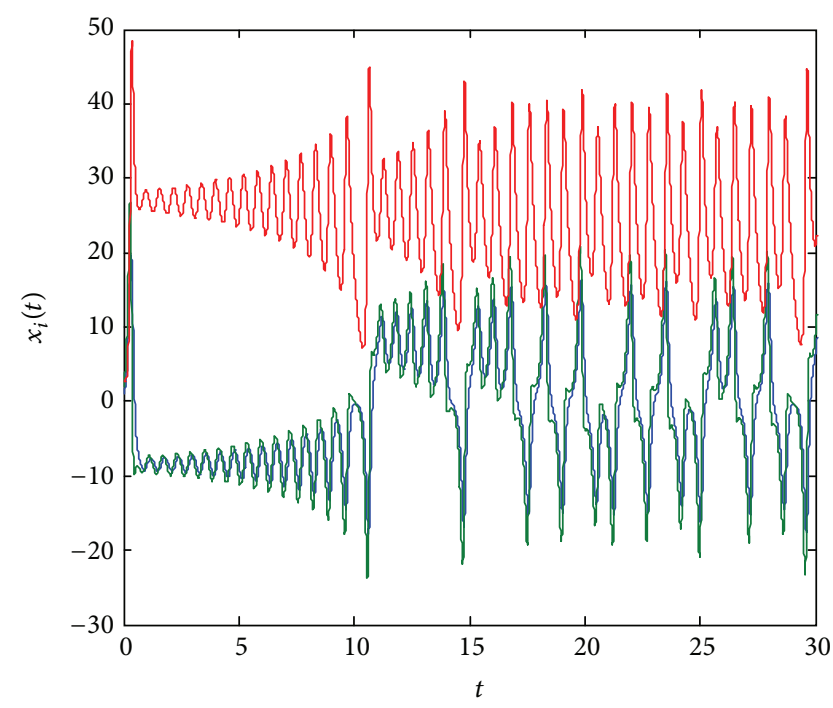

(b)

Figure 3: The node's dynamic in the second community.

The node dynamics of the second community as chaotic Lorenz system

$$
\begin{aligned}
& \dot{x}_{i 1}=10\left(x_{i 2}-x_{i 1}\right) \\
& \dot{x}_{i 2}=28 x_{i 1}-x_{i 2}-x_{i 1} x_{i 3} \\
& \dot{x}_{i 3}=x_{i 1} x_{i 2}-\frac{8}{3 x_{i 3}},
\end{aligned}
$$

with $i=6,7, \ldots, 10$. Figures 2,3 , and 4 depict the node's dynamic in different communities, respectively.
The node dynamics of the third community as hyperchaotic Lorenz system with $i=11,12, \ldots, 16$

$$
\begin{aligned}
& \dot{x}_{i 1}=10\left(x_{i 2}-x_{i 1}\right)+x_{i 4} \\
& \dot{x}_{i 2}=28 x_{i 1}-x_{i 2}-x_{i 1} x_{i 3} \\
& \dot{x}_{i 3}=x_{i 1} x_{i 2}-\frac{8}{3 x_{i 3}} \\
& \dot{x}_{i 4}=1.3 x_{i 4}-x_{i 1} x_{i 3} .
\end{aligned}
$$

For simplicity, the outer and inner coupling matrices are as follows: 


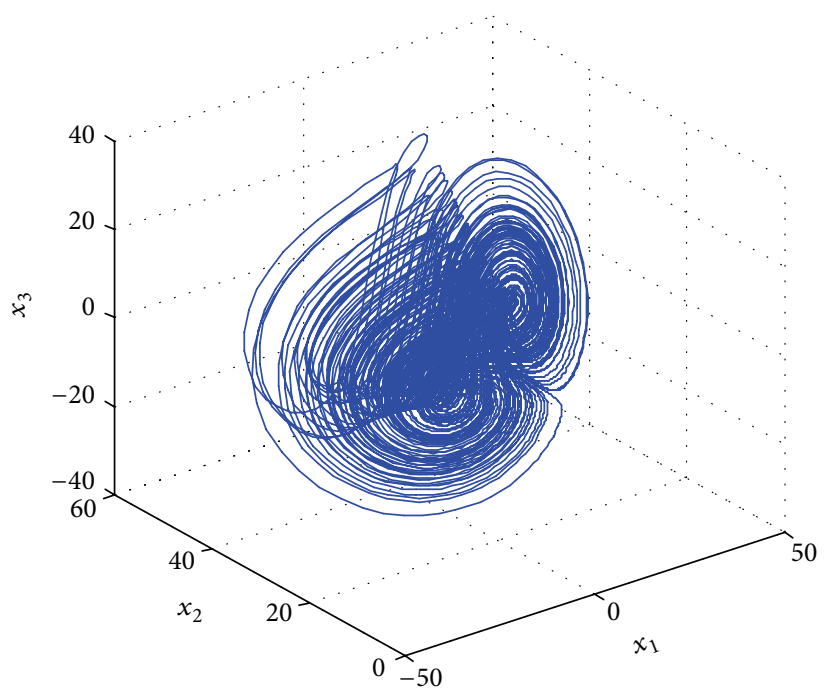

(a)

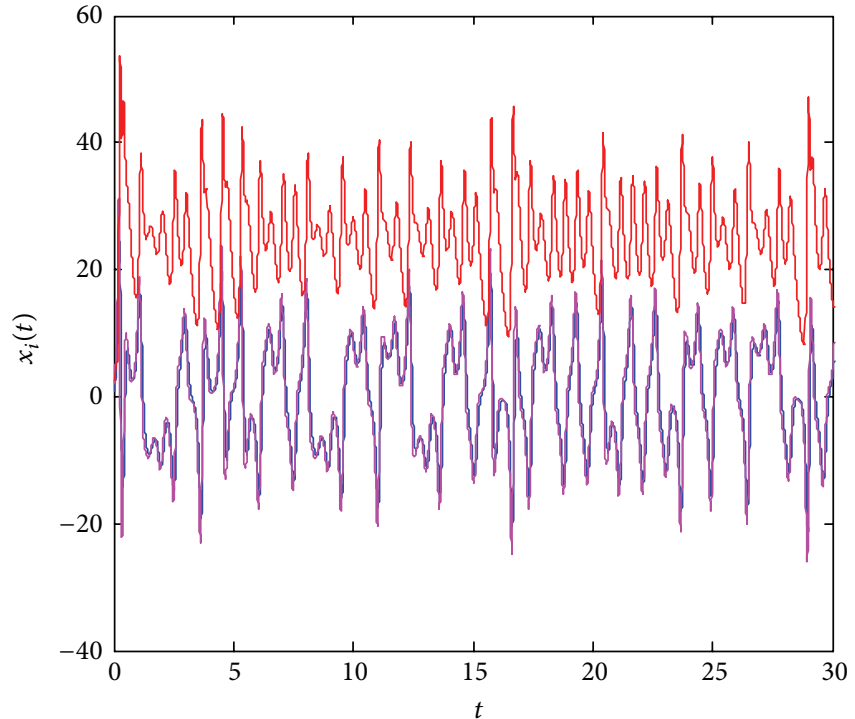

(b)

Figure 4: The node's dynamic in the third community.

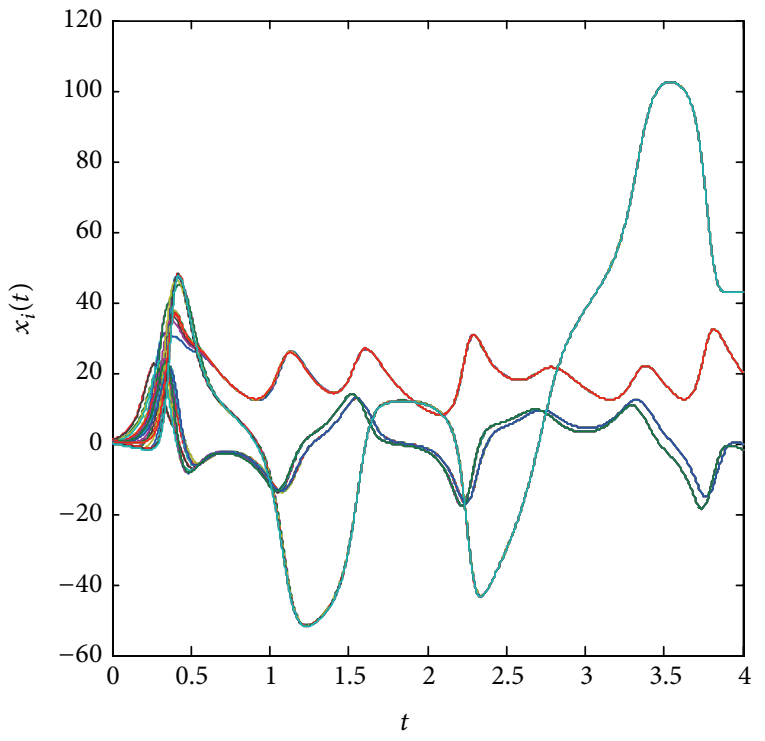

(a)

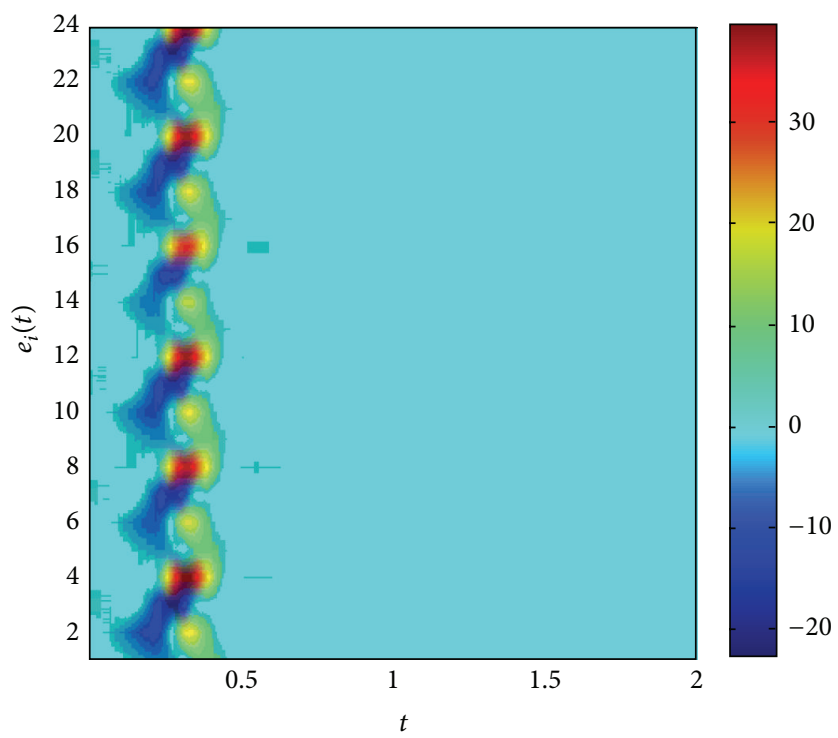

(b)

FIGURE 5: The orbits of state variable and synchronization errors in the first community.

(1) for nodes $i, j$ belong to the same community and there exist a connection between the nodes, then $a_{i j}=a_{j i}=$ 1

(2) $H_{11}=H_{33}=I_{4}, H_{22}=I_{3}$.

In numerical simulations, choose feedback gains $d_{i}=$ $80(i=1,2, \ldots, 18)$ and initial values of state variables $x_{i}(t)(i=1,2, \ldots, 18)$ randomly. Figures 5,6 , and 7 show the orbits of state variables and synchronization errors of all the communities, respectively.

\section{Conclusions}

In this paper, synchronization of a community network with different order node dynamics is investigated. Both adaptive feedback control and stability theory are adopted to design proper controllers. Adaptive feedback controllers were designed for achieving cluster synchronization based on the Lyapunov stability theory. Finally, numerical examples were provided to illustrate the effectiveness of the theoretical results. 


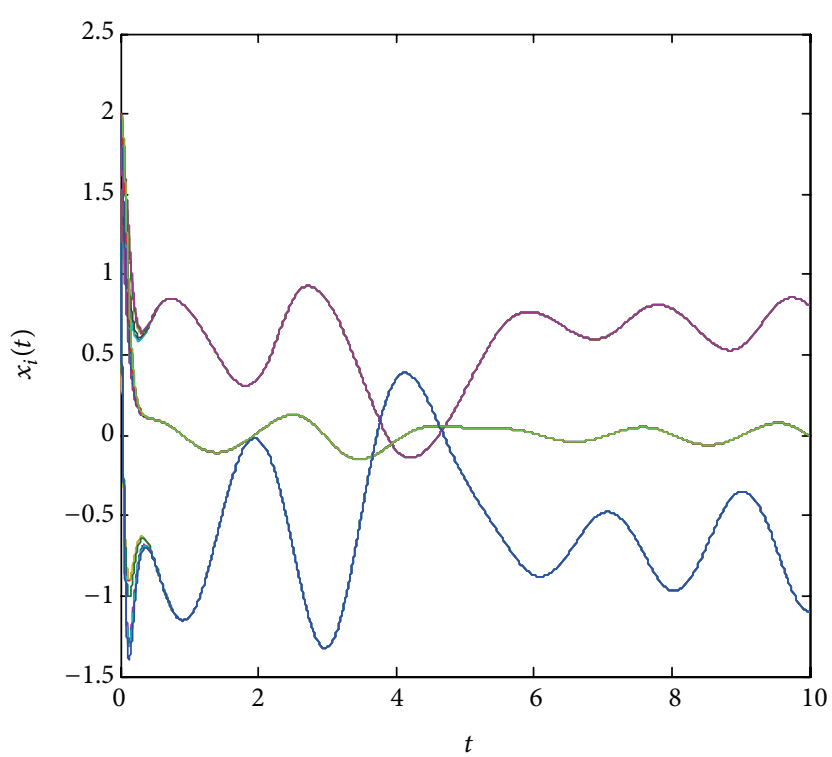

(a)

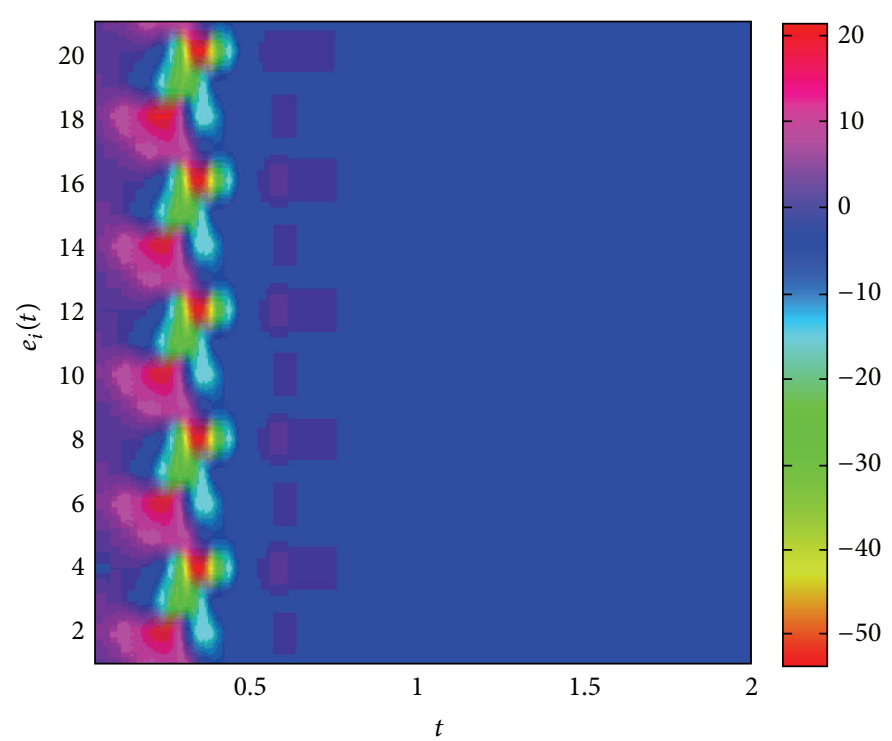

(b)

FIGURE 6: The orbits of state variable and synchronization errors in the second community.

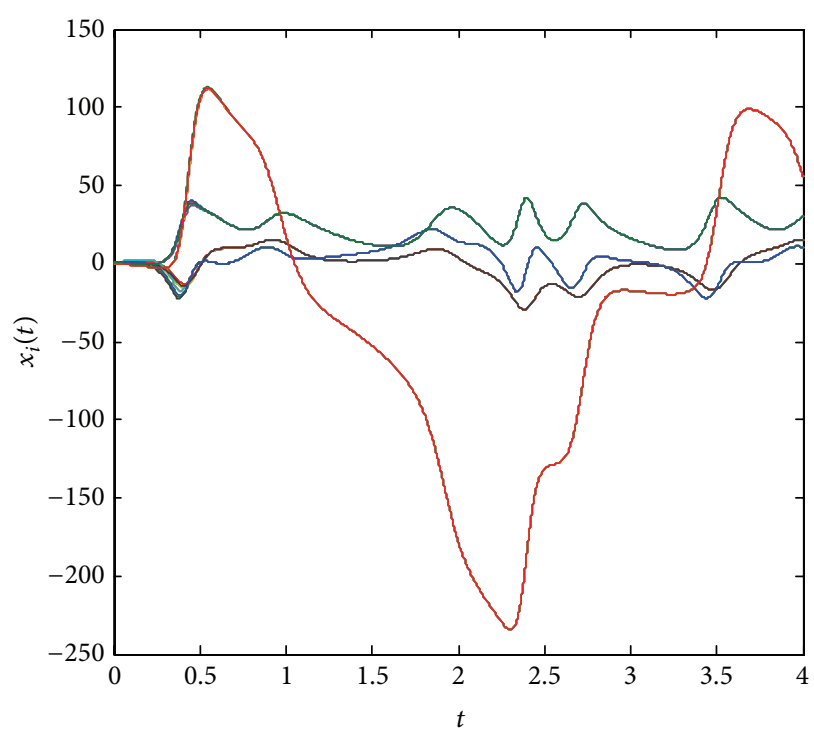

(a)

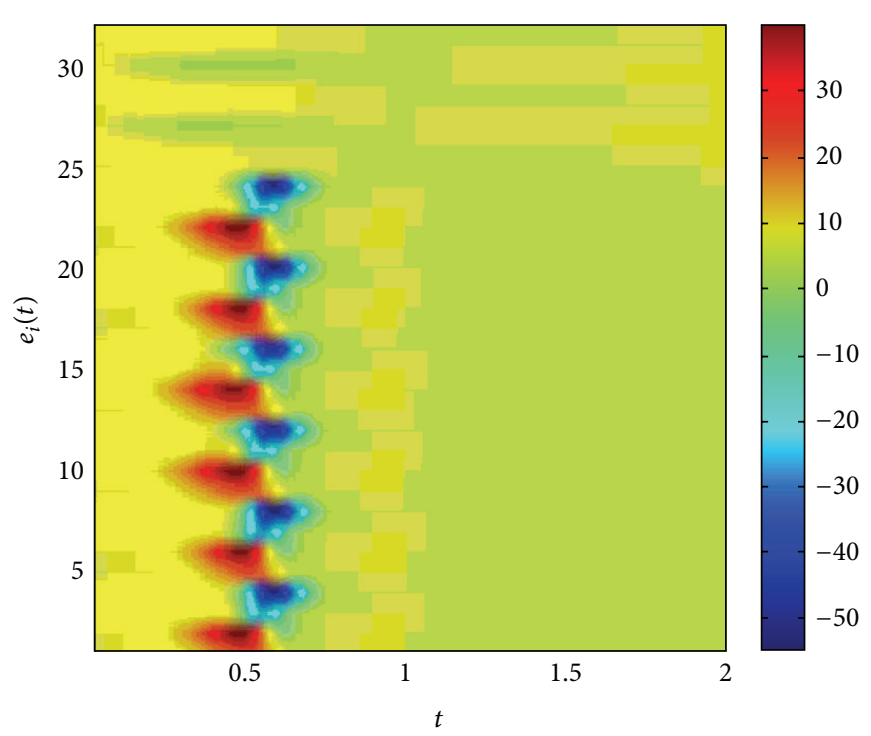

(b)

FIGURE 7: The orbits of state variable and synchronization errors in the third community.

\section{Conflict of Interests}

The authors declare that there is no conflict of interests regarding the publication of this paper.

\section{Acknowledgment}

The work was partially supported by Tianshui Normal University "QingLan" Talent Engineering Funds.

\section{References}

[1] S. H. Strogatz, "Exploring complex networks," Nature, vol. 410, no. 6825, pp. 268-276, 2001.

[2] A. B. Horne, T. C. Hodgman, H. D. Spence, and A. R. Dalby, "Constructing an enzyme-centric view of metabolism," Bioinformatics, vol. 20, no. 13, pp. 2050-2055, 2004.

[3] S. Boccaletti, V. Latora, Y. Moreno, M. Chavez, and D.-U. Hwang, "Complex networks: structure and dynamics," Physics Reports, vol. 424, no. 4-5, pp. 175-308, 2006. 
[4] M. L. Yang, Y. G. Liu, Z. S. You, and P. Sheng, "Global synchronization for directed complex networks," Nonlinear Analysis: Real World Applications, vol. 11, no. 3, pp. 2127-2135, 2010.

[5] J. Hu, Z. Wang, H. Dong, and H. Gao, "Recent advances on recursive filtering and sliding mode design for networked nonlinear stochastic systems: a survey," Mathematical Problems in Engineering, vol. 2013, Article ID 646059, 12 pages, 2013.

[6] J. Lü, X. Yu, and G. Chen, "Chaos synchronization of general complex dynamical networks," Physica A, vol. 334, no. 1-2, pp. 281-302, 2004.

[7] X. Wang and G. Chen, "Synchronization in small-world dynamical networks," International Journal of Bifurcation and Chaos, vol. 12, no. 5, pp. 187-192, 2002.

[8] M. Yang, Y. Liu, Z. You, and P. Sheng, "Global synchronization for directed complex networks," Nonlinear Analysis: Real World Applications, vol. 11, no. 3, pp. 2127-2135, 2010.

[9] G. Q. Si, Z. Y. Sun, Y. B. Zhang, and W. Q. Chen, "Projective synchronization of different fractional-order chaotic systems with non-identical orders," Nonlinear Analysis: Real World Applications, vol. 13, no. 4, pp. 1761-1771, 2012.

[10] M. Hu, Y. Yang, Z. Xu, R. Zhang, and L. Guo, "Projective synchronization in drive-response dynamical networks," Physica A: Statistical Mechanics and its Applications, vol. 381, no. 1-2, pp. 457-466, 2007.

[11] X. J. Wu and H. T. Lu, "Generalized function projective (lag, anticipated and complete) synchronization between two different complex networks with nonidentical nodes," Communications in Nonlinear Science and Numerical Simulation, vol. 17, no. 7, pp. 3005-3021, 2012.

[12] J.-W. Wang, Q. Ma, L. Zeng, and M. S. Abd-Elouahab, "Mixed outer synchronization of coupled complex networks with timevarying coupling delay," Chaos, vol. 21, no. 1, Article ID 013121, 2011.

[13] X.-Y. Wang and J.-M. Song, "Synchronization of the fractional order hyperchaos Lorenz systems with activation feedback control," Communications in Nonlinear Science and Numerical Simulation, vol. 14, no. 8, pp. 3351-3357, 2009.

[14] L.-X. Yang and J. Jiang, "Adaptive synchronization of driveresponse fractional-order complex dynamical networks with uncertain parameters," Communications in Nonlinear Science and Numerical Simulation, vol. 19, no. 5, pp. 1496-1506, 2014.

[15] Z. Y. Wu and X. C. Fu, "Cluster mixed synchronization via pinning control and adaptive coupling strength in community networks with nonidentical nodes," Communications in Nonlinear Science and Numerical Simulation, vol. 17, no. 4, pp. 16281636, 2012.

[16] W. Lu, B. Liu, and T. Chen, "Cluster synchronization in networks of coupled nonidentical dynamical systems," Chaos, vol. 20, no. 1, Article ID 013120, 12 pages, 2010.

[17] C. Hu and H. Jiang, "Cluster synchronization for directed community networks via pinning partial schemes," Chaos, Solitons \& Fractals, vol. 45, no. 11, pp. 1368-1377, 2012. 


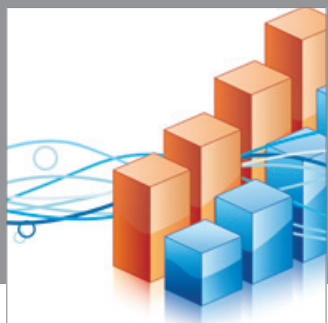

Advances in

Operations Research

mansans

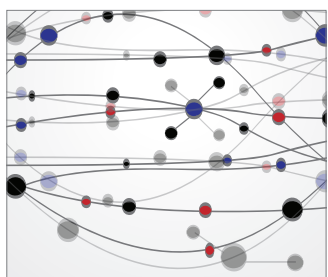

The Scientific World Journal
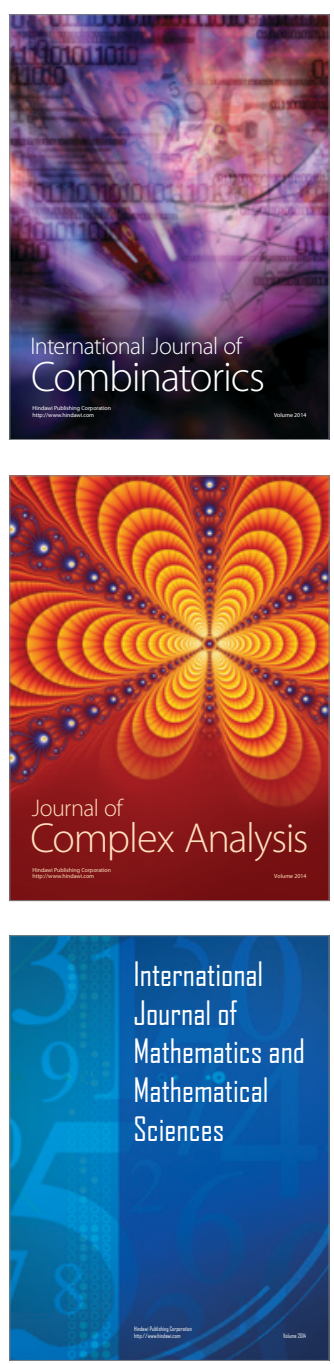
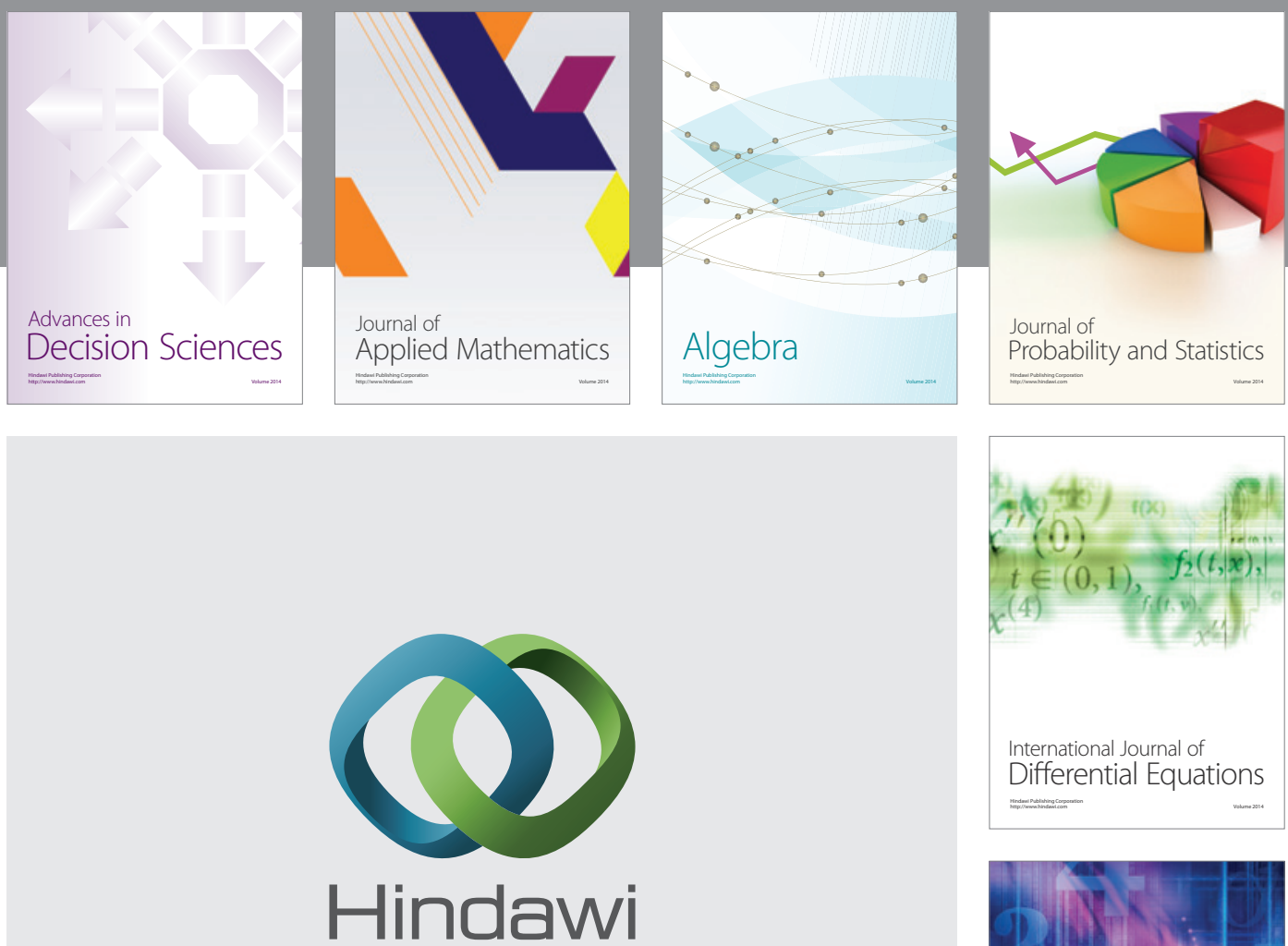

Submit your manuscripts at http://www.hindawi.com
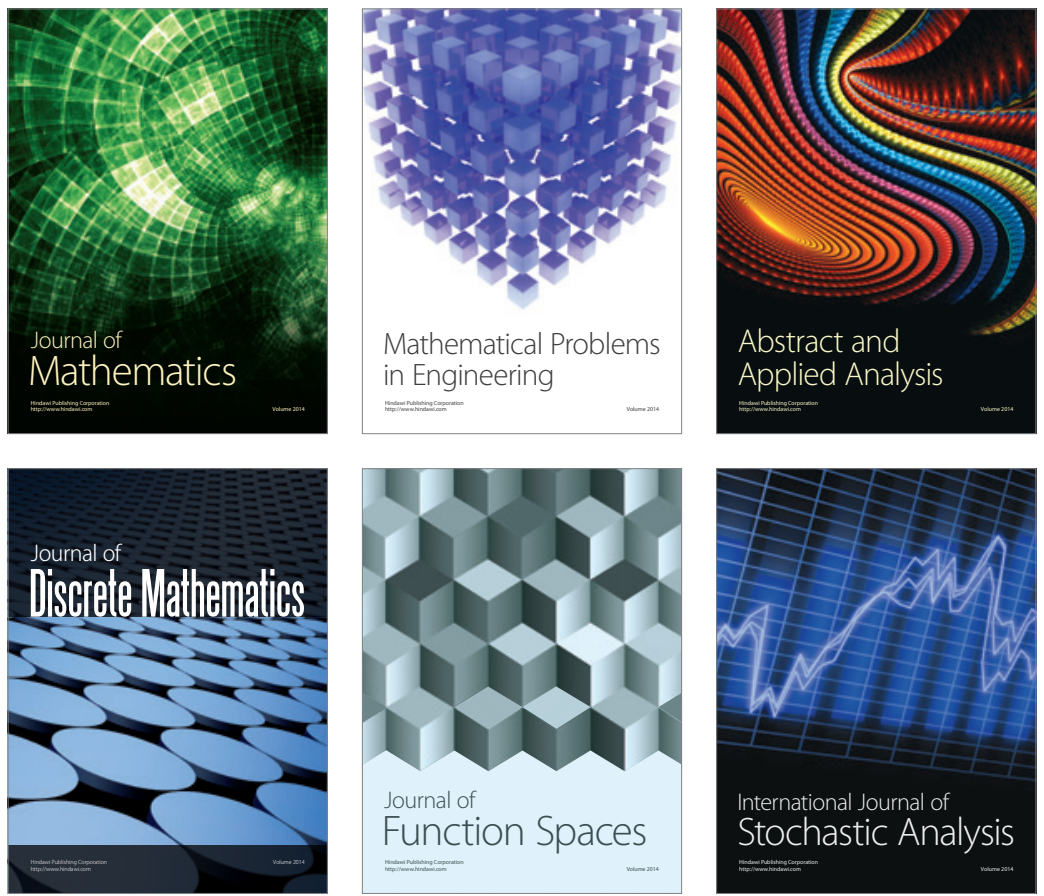

Journal of

Function Spaces

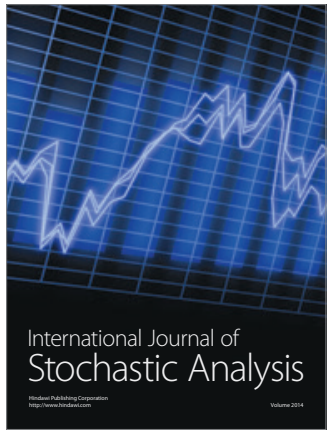

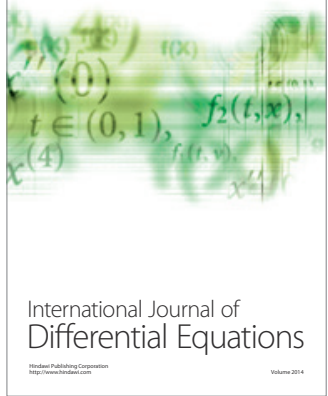
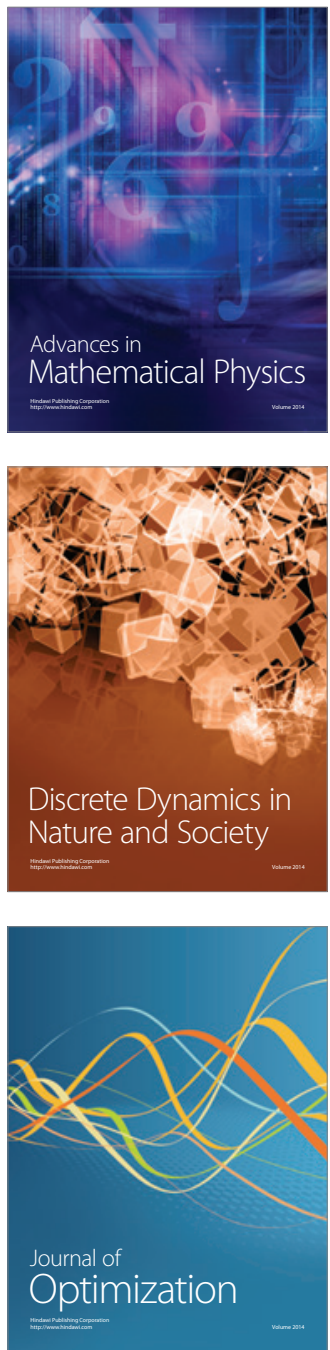